\title{
Aplicación de Flipped Classroom en la Carrera de Economía Agrícola: Una experiencia práctica
}

\section{Application of the Flipped Classroom in the Agricultural Economics Program: A practical experience}

\author{
MSc, Silvia Beatriz García Estupiñán ${ }^{1}$ \\ Sgarciauagraria@ug.edu.ec \\ MSc, Narcisa Cecilia Castro Chávez ${ }^{2}$ \\ narcisa.castrocug@ug.edu.ec \\ MSc, Francisco Mera Velasquez ${ }^{3}$ \\ Francisco.meravug@ug.edu.ec
}

Recibido: 1/09/2018; Aceptado: 1/11/2018

\begin{abstract}
RESUMEN
El aprendizaje y la enseñanza de un idioma exigen una interacción entre los alumnos y el docente en el salón de clases y una práctica real ya que se está tratando de una lengua y no una asignatura. El objetivo es proponer la utilización de la metodología del aula invertida en la clase de inglés para la carrera de Economía Agrícola, como alternativa de la enseñanza tradicional. La metodología consiste en un estudio de carácter cuantitativo, comparativo, por medio de grupos de control de las carreras economía agrícola con la estrategia de aula invertida y ciencias económicas; método tradicional, que en este caso es la población, tomando una muestra aleatoria. Se evaluó a los alumnos del IV semestre del periodo 2017-2018. La conclusión es optimizar el tiempo presencial en el salón de clases, tan necesario en la enseñanza de idiomas. Además, utilizar todo el tiempo presencial en la práctica real de la lengua con lo que se logró mejorar el rendimiento y competencia comunicativa.
\end{abstract}

Palabras Clave: aula invertida, aprendizaje, Inglés, estrategia, educación superior

\footnotetext{
${ }^{1}$ Universidad Agraria del Ecuador, Guayaquil, Ecuador.

2 Universidad Agraria del Ecuador, Guayaquil, Ecuador.

3 Universidad Agraria del Ecuador, Guayaquil, Ecuador.
}

Revista Científica Ciencia y Tecnología Vol. 19 No 21 págs. 65-76

http://cienciaytecnologia.uteg.edu.ec 


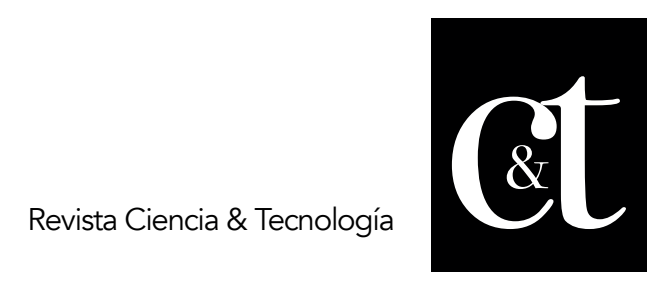

No. 21, 31 de enero de 2019

ISSN impreso: 1390 - 6321

\begin{abstract}
The learning and the teaching of a language require an interaction between the students and the teacher in the classroom a real practice since they are dealing with a language and not a subject. The objective is to propose the use of the methodology of the classroom invested in the English class for the degree of Agricultural Economics, as an alternative to traditional teaching. The methodology is quantitative, comparative, through control groups of the agricultural economy careers with the inverted classroom strategy and economic sciences. The traditional method, which in this case, a random sample of the population are students of the IV semester of the period 2017-2018. The conclusion: optimize classroom time is necessary in the teaching of languages. In addition, to use all the face-to-face time in the actual practice of the language with which it was possible to improve the performance and communicative competence.
\end{abstract}

Keywords: inverted classroom, learning, English, strategy, higher education

\title{
Introducción
}

Los constantes cambios que ha venido sufriendo la educación superior en el Ecuador ha evidenciado la necesidad de establecer políticas en la enseñanza de un idioma extranjero, que permitan tener competitividad a nivel internacional además del intercambio del conocimiento y una mayor movilidad entre instituciones. (Superior, Régimen Académico, 2008).

A lo que se deben tener en cuenta que el aprendizaje de idiomas requiere una interacción entre los alumnos y el docente, ya que el salón de clases es el único lugar que permite la práctica real de los aspectos comunicativos de la lengua, lo cual es necesario e importante utilizar métodos que mejoren el aprendizaje en el poco tiempo que se dispone para la enseñanza en la modalidad presencial. El flipped classroom (aula invertida) aparece como una nueva forma desafiando los métodos tradicionales.

El docente deberá cambiar su rol por las nuevas exigencias de la sociedad en la cual este deberá ser un mediador del conocimiento y planificador del aprendizaje, actuando como apoyo para favorecer la construcción del pensamiento por parte del estudiante. Investido en este nuevo rol el docente está obligado sobre su propia práctica educativa para elaborar nuevas propuestas pedagógicas adecuadas a los nuevos retos que se presentan en la sociedad del conocimiento y así como surge esta nueva forma de enseñar que se llama Flipped Classroom o aula invertida.

La metodología de Flipped Classroom se basa en utilizar el tiempo disponible del alumno fuera del aula, realizando determinados procesos que normalmente se hacen en clases lo pueden hacer fuera de ella y en clase potenciar y facilitar la práctica del conocimiento en el aula (Sánchez, 2013).

En un aula de método tradicional el profesor dicta o imparte la clase como una conferencia y el alumno escucha intentando comprender y aprender lo que ahí se dice, cuando en casa se puede hacer esa tarea, es decir, leer el tema a tratarse en la clase siguiente.

\section{Desarrollo}




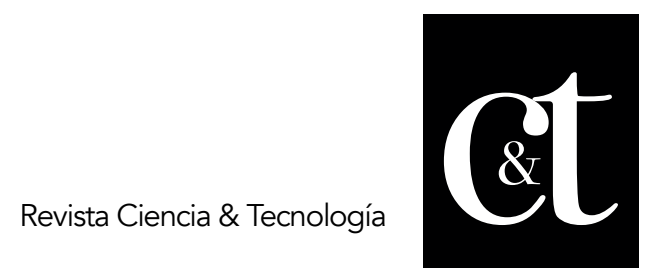

No. 21, 31 de enero de 2019

ISSN impreso: 1390 - 6321

Siendo el punto clave es que fuera del aula los estudiantes trabaja en forma anticipada el material que van a tratar en clases mediante material audio visual y explicativo, la idea de Aula Invertida va más allá, es decir, que el alumno llegue al salón de clases con conceptos revisados y en clases realizar talleres que complementan y afirman esos conceptos, procedimientos, de tal manera que se instituye como un enfoque integral que da paso a la transmisión de conocimiento y mejora la interacción entre los alumnos y el profesor creando entornos de aprendizaje en donde el estudiante se convierte en responsable de su propio aprendizaje . (Ruiz, N., Mendoza, M. y Ferrer, L. (2013).

Con esta nueva metodología de aula invertida se cambia el modelo de trabajo y se invierten los papeles de una clase tradicional gracias al apoyo de las tecnologías de la información y la comunicación. (TIC). Teniendo los estudiantes la oportunidad de revisar el material en línea las veces necesarias y por otro lado el docente en clases interviene como guía de las actividades prácticas dinámicas, creando un ambiente para el debate y exponer sus ideas lo cual exige la escuela nueva o el aprendizaje significativo (Pérez, 2015).

En este proyecto se presenta una propuesta de Aplicación de la Aula Invertida en la asignatura de Ingles IV para la Carrera de Economía Agrícola de la Facultad de Economía Agrícola de la Universidad Agraria del Ecuador en la ciudad de Guayaquil. La propuesta se implementó en el período académico 2017-2018 para la asignatura de inglés IV para la Carrera de Economía Agrícola y la Carrera de Ciencias Económicas del Cuarto Semestre de la Facultad de Economía Agrícola, con una carga de 5 créditos.

El proyecto combina la enseñanza tradicional a los alumnos de la Carrera de Ciencias Económicas y la metodología de flipped classroom o aula invertida a los de Economía Agrícola dándoles la posibilidad de practicar en casa aspectos léxicos a través de distintos materiales tantos de elaboración propia subidos a la plataforma de la universidad como actividades interactivas y audiovisuales disponibles en el texto o en el internet herramienta con información de primera y muy útil para motivación en el aprendizaje activo y autónomo. La introducción de la enseñanza inversa permite a los alumnos a trabajar a su propio ritmo según sus conocimientos, así como, la forma de afrontar el material proporcionado de acuerdo sus propios intereses personales (Shapiro, 2013).

Como se menciona en (Perdomo, 2016) "el modelo flipped classroom tiene un fuerte componente de responsabilidad y motivación por parte del estudiante para apropiar una información y transformarla en conocimiento a partir de la aplicación práctica en un aula de clase. Así, el estudiante desarrolla su aprendizaje a partir de diversas situaciones que facilitan u optimizan el mismo, tales como sus experiencias, intereses y motivaciones, las problemáticas asociadas y las alternativas de solución".

En este nuevo marco, lo elementos de la motivación, el trabajo autónomo, permite al docente y al estudiante innovar en las estrategias motivacionales que garanticen al alumno la apropiación del conocimiento y el modelo aula invertida ayuda al dicente que abarque contenidos formales por cuenta propia y tenga claro que su autoevaluación y autorreflexión son importantes en dicho proceso, dándole al 


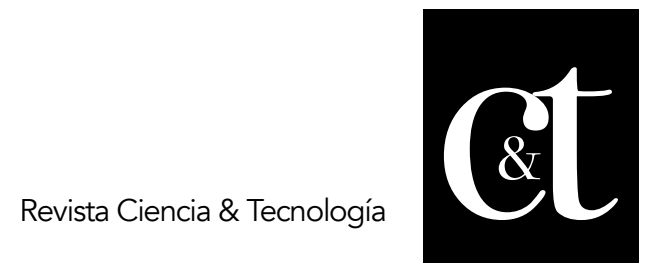

No. 21, 31 de enero de 2019

ISSN impreso: 1390 - 6321

maestro la oportunidad de guiar al alumno en forma óptima.

Con la aplicación de esta nueva estrategia se incrementó la motivación del aprendizaje del idioma inglés en los alumnos y mejorar su rendimiento académico, poniendo al servicio del aprendizaje activo las tecnologías y fomentando el desarrollo del aprendizaje autónomo. Esto les será de gran utilidad en la formación de su carrera y vida profesional, optimizando el poco tiempo presencial en el aula, tan necesario en la enseñanza de idiomas y empleando de mejor manera las distintas macro destrezas lingüísticas en la enseñanza de idiomas simulando en la práctica de situaciones que se aproximen a la vida real.

\section{Material y Método}

El proyecto se inicia con una prueba de diagnóstico a los grupos de control, que en este caso es la población tomando una muestra aleatoria. Se evaluó a los alumnos del IV semestre del periodo 2017-2018 de las carreras antes mencionadas. Después, se aplica el tratamiento a los dos grupos el de economía agrícola con la estrategia de aula invertida y el de ciencias económicas el método tradicional y finalmente se replica la misma prueba al final del semestre, teniendo como punto de referencia el puntaje obtenido al inicio del semestre y como variables la motivación y las tics en el aprendizaje activo con el aula invertida al grupo de economía agrícola, lo que el de ciencias económicas solo el uso del texto

En este artículo se redacta la experiencia obtenida con los grupos pilotos de la Facultad de Economía Agrícola en la asignatura de inglés IV, con la aplicación de la estrategia de aula invertida y sus resultados obtenidos. La experiencia tiene como finalidad fomentar en los alumnos el aprendizaje activo dentro y fuera del aula, de forma que puedan llegar a los salones de clases con un material previamente revisado y entendido y con mayor motivación o disposición de resolver actividades planteadas y exponer sus puntos de vista en donde se está creando estudiantes críticos y reflexivos.

Para esto, los alumnos deben organizar y planificar su dedicación a la materia de inglés y los docentes deben seleccionar los contenidos, desarrollar los recursos y realizar una organización, programación y planificación en forma óptima. Se seleccionó videos y bibliografía relevante existentes en el internet en base al syllabus establecido para la asignatura de inglés IV de los diferentes contenidos en las carreras de economía agrícola y ciencias económicas. De todo lo encontrado, se seleccionaron aquellos videos que contengan exposición clara y amena, quedando a disposición de los estudiantes en la plataforma de la universidad.

Descripción de la experiencia - Desarrollo

La aplicación del método Flipped Clasroom permite enfocar la atención en la búsqueda, determinación e interpretación y aplicación del conocimiento desde una perspectiva autónoma y poder llegar al aula y socializar la información para poder contrarrestarla. La educación superior al aplicar estos métodos, estrategias y técnicas innovadoras incentivan en el estudiante la reflexión, el trabajo autónomo, motivar su propio quehacer en el estudiante y en el docente reafirmar o reestructurar sus concepciones y horizontes pedagógicos en la aplicación de las metodologías creativas y la potencialización y generación del aprendizaje significativo. Según (Martí Parreño,

68 


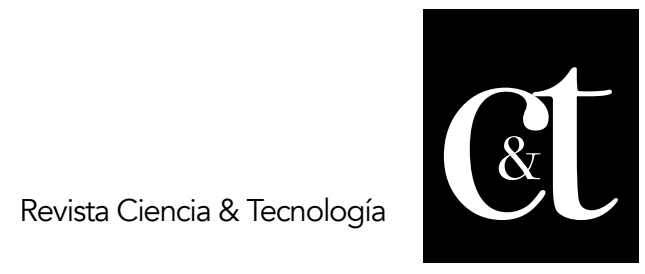

No. 21, 31 de enero de 2019

ISSN impreso: 1390 - 6321

Prado Gascó, Queiro Ameijeiras, \& Summerfield, 2014), entre las principales características de la flipped classroom se encuentra la utilización de un mayor tiempo en el aula para la aplicación práctica de conocimientos teóricos previamente adquiridos mediante procesos de autoaprendizaje fuera del aula. Para llevar a cabo el proyecto en práctica, se conversó con los estudiantes sobre lo que era flipped classroom y los beneficios que obtendrían con la aplicación en el aprendizaje del idioma inglés, con días de anticipación para que tengan conocimientos de las actividades a realizarse dentro y fuera del aula, entregándoles una guía de cada actividad. En dicha guía de la actividad, se especificaban claramente las competencias específicas que el alumno debía adquirir y en el aula se remarcó la importancia de dichas competencias, ya que supone el entendimiento o no del tema trabajado.

La asignatura de inglés se imparte en los ocho semestres de las Carreras de Economía Agrícola y Ciencias Económicas, con una carga horaria de cinco horas semanales, que corresponde a cinco créditos a la malla curricular, lo cual es una de las asignaturas que da mayor peso a la misma. En el Ciclo I del año lectivo 20172018, de forma presencial se puso en marcha la práctica del uso de la metodología del Flipped Classroom (aula invertida) con dos grupos de cuarto semestre, respectivamente uno con la metodología antes mencionada y el otro con la metodología tradicional.

A los estudiantes que se les aplicó la nueva estrategia metodológica. Realizaron en sus horas autónomas la tarea de trabajar con los videos tomados de la plataforma de YouTube en el internet con temas de gramática específicamente y temas de motivación. La conversación, con la preparación del vocabulario y las horas presenciales, sirvieron para aclarar dudas y crear un ambiente de comunicación entre ellos ya que uno de las habilidades menos desarrolladas fue el speaking, por el temor a hablar, equivocarse y sentirse parte de burla por parte de sus compañeros. El flipped classroom les ayudaba a crear un vocabulario, dar ideas y poder desarrollarlas en clase de inglés hablando en inglés. La asignatura se divide en cuatro bloques de estudio con ocho unidades, habiendo elaborado una sesión de aula invertida con gramática y temas de conversación incluyendo lecturas con temas de su interés que sirvan para fomentar el debate.

El tiempo que el alumno utilizó para las secciones del flipped classroom varía según el nivel de conocimiento de cada uno, pero la duración prevista fue de 4 horas autónomas, las cuales se distribuyeron a lo largo de dos semanas. Por lo tanto, en las horas presenciales se revisó el vocabulario y expresiones más importantes consolidando puntos gramaticales, lo que le permite al estudiante las destrezas comunicativas y mejorar el aprendizaje del idioma de lengua extranjera (inglés), llegando a concretar clases más interactivas y prácticas

\section{Resultados}

Para apreciar el resultado de la experiencia se debe tomar en cuenta la percepción del docente y del estudiante. La valoración por parte del docente fue muy relevante. El esfuerzo en la búsqueda de materiales audiovisuales específicos obtenidos de YouTube y el diseño de actividades propuestas para realizar en el aula envuelven una metodología que supone una renovación a la hora de enseñar y permite profundizar en los contenidos. 


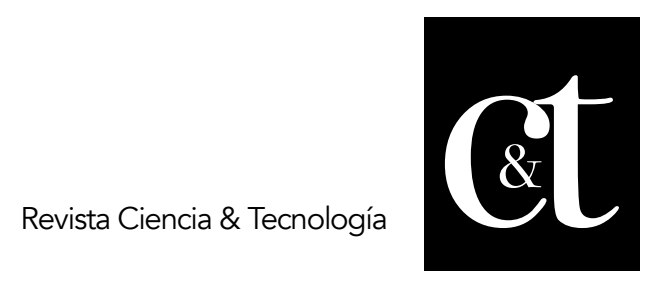

No. 21, 31 de enero de 2019

ISSN impreso: 1390 - 6321

En el ciclo I del periodo 2017-2018, se contaba con 10 semestres de ambas carreras de Economía Agrícola y Ciencias Económicas, siendo los grupos pilotos los alumnos del 4to semestre, respectivamente con 40 alumnos por semestre. En el uno se aplicó el Flipped classroom y en otro se manejó el método tradicional. De antemano, se le comunicó a los alumnos de la práctica que se iba a implementar con ellos para que manejen su tiempo de dedicación a las tareas y revisión de material extra. Ambos grupos recibieron el mismo syllabus la variación se dio en la forma que recibieron las clases, lo que a se manifestó anteriormente.

Para evaluar esta experiencia de innovación se inició con un pre test de diagnóstico a los dos grupos y después del término del semestre se los volvió a evaluar con un post test para medir el grado de rendimiento académico. También se realizaron encuestas de la experiencia con el fllipped classroom, para así analizar el grado de consecución de los objetivos propuestos.

De este modo, por un lado, se analizó los resultados de la encuesta que se realizó en forma anónima, con las que los alumnos valoraron la organización, planificación y la metodología del trabajo, los materiales didácticos desarrollados el uso de las TIC y la evaluación de los aprendizajes. Además, se cuestionó los aspectos de la innovación que sería necesario para modificar su mejora. A continuación, se analizaron los resultados más relevantes de la evaluación de la experiencia.

\section{Tabla 1. Muestra}

\begin{tabular}{|l|l|l|}
\hline Muestra Estudiantes & $\%$ \\
\hline $\begin{array}{l}\text { Carrera de Economía } \\
\text { Agrícola IV Semestre }\end{array}$ & 40 & $50 \%$ \\
\hline $\begin{array}{l}\text { Carrera de Ciencias } \\
\text { Económicas IV Semestre }\end{array}$ & 40 & $50 \%$ \\
\hline Total & 80 & $100 \%$ \\
\hline
\end{tabular}

Fuente: Universidad Agraria del Ecuador

Tabla 2. Cuadro de la escala de evaluación para el estudio

\begin{tabular}{|l|l|}
\hline Escala & Equivalencia \\
\hline $10-7$ & Muy Bueno \\
\hline $6-5$ & Bueno \\
\hline 4 o menos & Insuficiente \\
\hline
\end{tabular}

Fuente: Universidad Agraria del Ecuador 
Tabla 3. Rango de Calificaciones del pretest de Economía Agrícola

\begin{tabular}{|l|l|l|l|}
\hline Carrera & N. de Estudiantes & Calificaciones & Porcentaje \\
\hline \multirow{2}{*}{$\begin{array}{l}\text { Economía } \\
\text { Agrícola }\end{array}$} & 10 & 10 a 7 & $25 \%$ \\
\cline { 2 - 4 } & 12 & 6 a 5 & $30 \%$ \\
\cline { 2 - 4 } & 18 & 4 a menos & $45 \%$ \\
\hline Total & 40 & & $100 \%$ \\
\hline
\end{tabular}

Fuente: Universidad Agraria del Ecuador

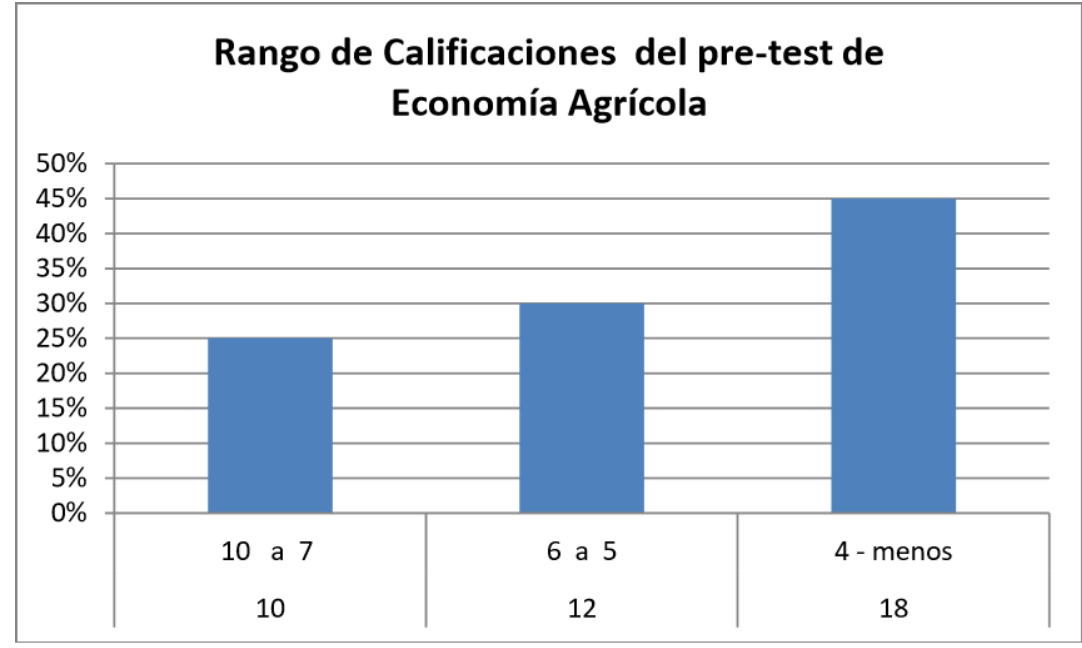

Gráfico 1. Rango de calificaciones del pretest de Economía Agrícola Fuente: Universidad Agraria del Ecuador

Tabla 4. Rango de Calificaciones del pretest de Ciencias Económicas

\begin{tabular}{|l|l|l|l|}
\hline Carrera & N. de Estudiantes & Calificaciones & Porcentaje \\
\hline Ciencias & 8 & 10 a 7 & $20 \%$ \\
\cline { 2 - 4 } Económicas & 15 & 6 a 5 & $37.5 \%$ \\
\cline { 2 - 4 } & 17 & 4 a menos & $42.5 \%$ \\
\hline Total & 40 & & $100 \%$ \\
\hline
\end{tabular}

Fuente: Universidad Agraria del Ecuador 


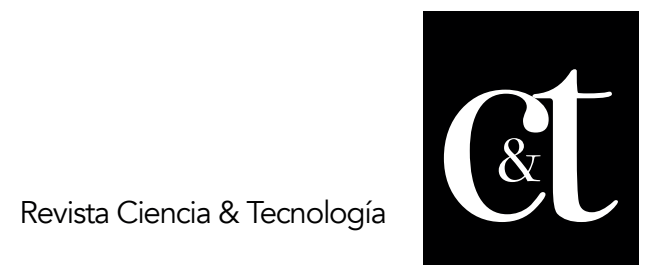

No. 21, 31 de enero de 2019

ISSN impreso: 1390 - 6321

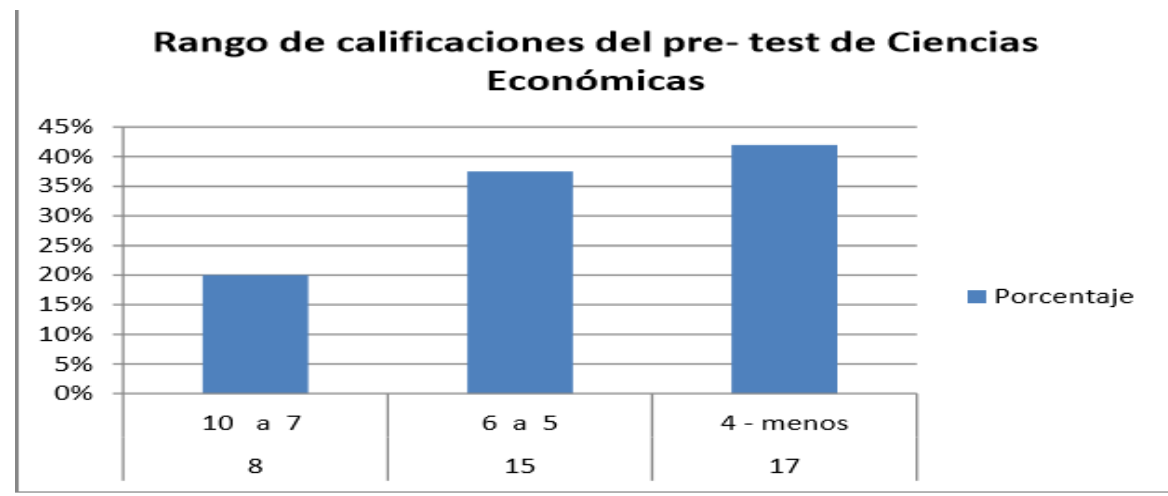

Gráfico 2. Rango de calificaciones del pretest de Ciencias Económicas Fuente: Universidad agraria del Ecuador

Tabla 5. Rango de Calificaciones del postista de Economía Agrícola

\begin{tabular}{|l|l|l|l|}
\hline Carrera & N. de Estudiantes & Calificaciones & Porcentaje \\
\hline \multirow{2}{*}{$\begin{array}{l}\text { Economía } \\
\text { Agrícola }\end{array}$} & 25 & 10 a 7 & $63 \%$ \\
\cline { 2 - 4 } & 10 & 6 a 5 & $25 \%$ \\
\cline { 2 - 4 } & 5 & 4 a menos & $12 \%$ \\
\hline Total & 40 & & $100 \%$ \\
\hline
\end{tabular}

Fuente: Universidad agraria del Ecuador

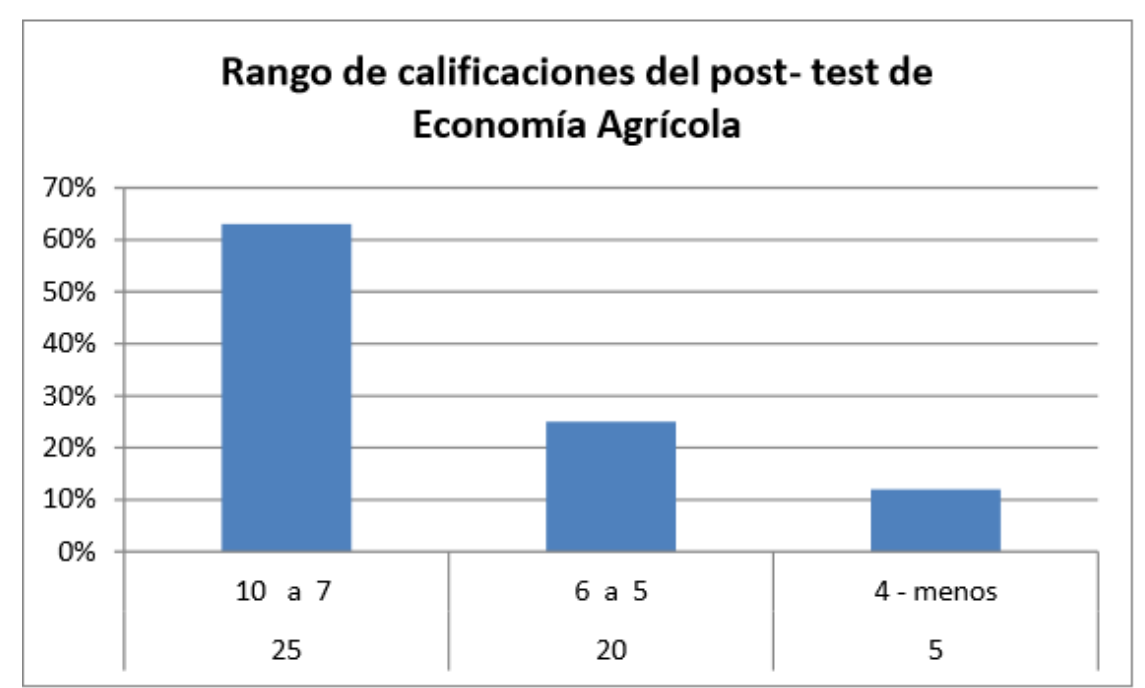

Gráfico 3. Rango calificaciones post-test de Economía Agrícola Fuente: Universidad Agraria del Ecuador 
Respeto al análisis cuantitativo en la prueba pre - test de ambas carreras se puede destacar la realidad de los conocimientos de los alumnos en base al rango de calificaciones.

Tabla 6. Rango de calificaciones del post-test de Ciencias Económicas

\begin{tabular}{|l|l|l|l|l|}
\hline \multicolumn{2}{|l|}{ Carrera } & $\begin{array}{l}\text { N. } \\
\text { Estudiantes }\end{array}$ & Calificaciones & Porcentaje \\
\hline & 8 & 10 a 7 & $20 \%$ \\
\hline \hline $\begin{array}{l}\text { Ciencias } \\
\text { Económicas }\end{array}$ & 20 & 6 a 5 & $50 \%$ \\
\cline { 2 - 5 } & 12 & 4 a menos & $30 \%$ \\
\hline Total & 40 & & $100 \%$ \\
\hline
\end{tabular}

Fuente: Universidad agraria del Ecuador

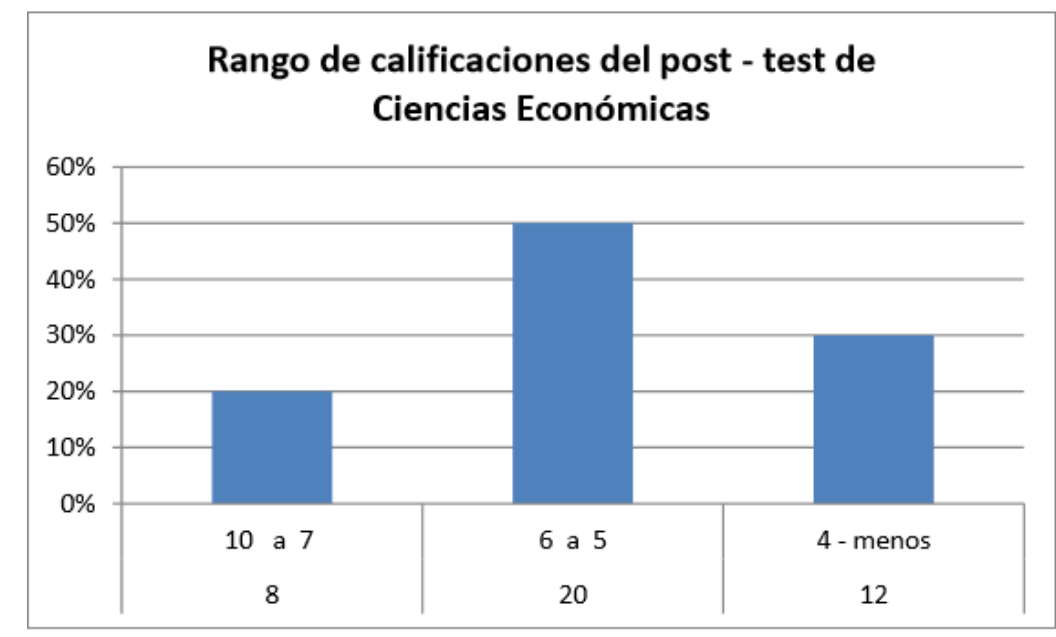

\section{Gráfico 4. Rango calificaciones post test de Economía Agrícola}

Fuente: Universidad Agraria del Ecuador

Hay una diferencia representativa en las medidas de calificaciones de los estudiantes antes y después del uso de la metodología Flipped Classroom de la asignatura de inglés en los cuartos semestres, por lo cual se considera que la metodología de aula invertida $\mathrm{Si}$ tiene resultados significativos en los resultados del rendimiento académico. Lo cual se puede observar en los cuadros estadísticos con esto se 
demuestra que sería bueno aplicar esta nueva metodología y dejar (Ecuador, 2017) de impartir una clase mucho más en la educación superior

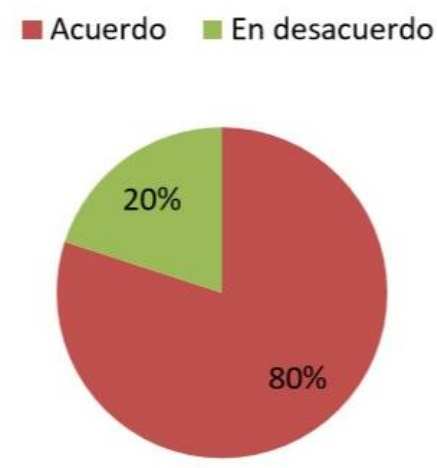

\section{Gráfico 5. Uso del flipped classroom en la carrera de Economía Agrícola Fuente: Universidad Agraria del Ecuador}

En cuanto a los resultados a los resultados de las encuestas, se pudo obtener la siguiente información: un $80 \%$ de los alumnos que utilizaron el flipped classroom por ser una metodología nueva, ya que se motivaron a aprender más el idioma, por lo muchos de ellos lo consideraban una materia más y como futuros economistas, no estimaban que podía ser útil en su campo laboral y a través del aula invertida vieron una posibilidad de mejorar en su nivel del idioma extranjero, por lo que lo recomiendan que sea aplicado en otras asignaturas y el $20 \%$ que no estuvo de acuerdo por no contar con el tiempo para trabajar los videos enviados a casa por ser estudiantes que trabajan a pesar de cursar en un sistema presencial, prefieren recibir todo en clases lo que quiere decir que optan por un modelo tradicional, ya que los obliga a trabajar en una forma que no estaban acostumbrados.

\section{Conclusiones}

Es necesario introducir nuevas estrategias o metodologías que motiven al aprendizaje activo. El uso del flipped classroom o aula invertida que utiliza las TIC's con los sistemas tradicionales, mejora el rendimiento académico. En donde esta nueva propuesta aplicada optimiza el tiempo asignado en los syllabus en la asignatura de inglés en la Facultad de Economía Agrícola de modo que los estudiantes podrían alcanzar el nivel deseado en el momento de obtener su grado.

Se puede deducir que en educación no hay procedimientos mágicos que se ajusten todas las circunstancias, sino que se trata de aprovechar las potencialidades de todos los recursos, para así alcanzar nuestros objetivos." Las clases invertidas representan una posibilidad más que la tecnología pone a nuestro alcance, una ayuda para que el profesor se convierta en un facilitador del aprendizaje, una visión a tener en cuenta 
puede resultar particularmente útil para el conocimiento de idiomas ya que la mejor forma de adquirir competencias lingüísticas es a través del desarrollo de estrategias comunicativas" (Sánchez, Ruiz y Sánchez, 2014).

Derivando de la percepción de alumnos respecto a la metodología y resultados de aprendizaje del curso, es interesante que, en la mayoría de los resultados de la encuesta, en una pregunta en particular arrojara 100\%, presumiblemente los alumnos consideraron pertinente la estrategia de aprendizaje que los videos académicos apoyarían y complementarían la explicación de las clases en los alumnos volviéndola más entendible e interactiva, los alumnos sugirieron que deberían emplear siempre herramientas para poder ayudar a su enseñanza.

Los alumnos actualmente están acostumbrados a buscar información en forma digital y muchas veces prefieren estudiar fuera del aula tradicional. Para motivar tanto a los estudiantes más débiles, que necesitan más repetición, como a los más avanzados, que están dispuestos a tomar más responsabilidad y a determinar su propio ritmo de aprendizaje, es necesario adoptar nuevos métodos de enseñanza y aprendizaje.

Se logró cumplir con los objetivos determinados al inicio de la presente tesis, se realizó la producción de los recursos TIC para apoyar en el aprendizaje de los alumnos y se identificó los factores que influyen en la no utilización de la metodología Flipped Classroom.

\section{Referencias bibliográficas}

Consejo de Educación Superior. (2008). Reglamento de Régimen Académico. República del Ecuador. Quito, Ecuador.

Martí Parreño, J., Prado Gascó, V., Queiro Ameijeiras, C., \& Summerfield, Lynn, C. (2014). Principales barreras y facilitadores del uso de las flipped classrooms en el aula: una aproximación cualitativa. Universidad europea. Obtenido de http://abacus.universidadeuropea.es/handle/11268/3597

Perdomo. (2016). Estudio de evidencias de aprendizaje significativo en un aula bajo el modelo de flipped clasroom. Edutec. No. 55, pp. 1-17.

Pérez-Guillot, C. (2015). Flipped teaching aplicando al inglés para la gestión empresarial: una nueva experiencia. Valencia, España. Ed. U de Valencia.

Régimen Académico de Educación Superior (2008). Quito.

Ruiz, N., Mendoza, M. y Ferrer, L. (2014). Influencia de las tecnologías de información y comunicación de los roles e interrelaciones entre estudiantes y docentes en programas presenciales de educación superior. Hallazgos. V. 11 (22), pp. 435-454.

Sánchez (2017). Las clases invertidas: beneficios y estrategias para su puesta en práctica en la educación superior. Educación mediática y competencia digital. V. $6(2)$.

Sánchez, J., Ruiz, J., y Sánchez, E. (2014). Flipped classroom una experiencia abierta y flexible. Málaga, España: Ed. Universidad de Málaga. 


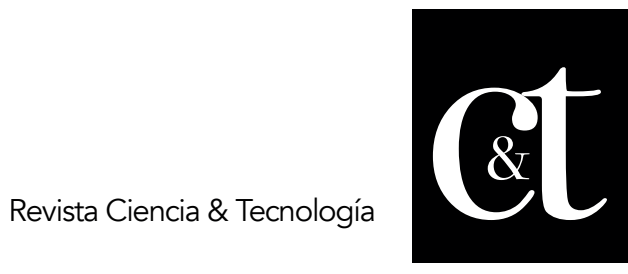

No. 21, 31 de enero de 2019

ISSN impreso: 1390 - 6321

Shapiro, L. (2013). Aula Invertida o Flipped Classroom - Fundamentos y Beneficios 17 Herramientas TIC para Trabajar el Aula Invertida. Recuperado de: https://gesvin.wordpress.com/2015/11/18/17-herramientas-tic-para-trabajarel-aula-invertida-articulo/ 